MATHEMATICAL ASSOCIATION

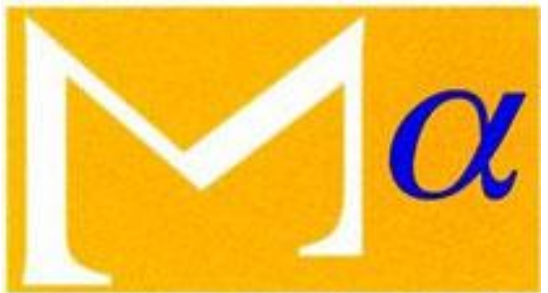

supporting mathematics in education

On a Problem in Mechanics and the Number of Its Solutions

Author(s): F. S. Macaulay

Source: The Mathematical Gazette, Vol. 3, No. 59 (Oct., 1906), pp. 365-373

Published by: Mathematical Association

Stable URL: http://www.jstor.org/stable/3603717

Accessed: 25-11-2015 14:33 UTC

Your use of the JSTOR archive indicates your acceptance of the Terms \& Conditions of Use, available at http://www.jstor.org/page/ info/about/policies/terms.jsp

JSTOR is a not-for-profit service that helps scholars, researchers, and students discover, use, and build upon a wide range of content in a trusted digital archive. We use information technology and tools to increase productivity and facilitate new forms of scholarship. For more information about JSTOR, please contact support@jstor.org. 
of detail is perhaps a little alarming, but there is only one dominating idea, which is not hard, for the whole theory. And the writer repeats that a knowledge of this theory is not for the immature schoolboy mind; very serious damage has been done to mathematical training by attempts to gloss over the inherent difficulties which lie at the foundations of the Higher Trigonometry.

D. K. PICKen.

\section{ON A PROBLEM IN MECHANICS AND THE NUMBER OF ITS SOLUTIONS.}

To find the position of equilibrium of a particle which is acted on by forces of given magnitudes directed towards given points.

This problem was suggested by Mr. P. A. Hillhouse, and arose in the first instance from the consideration of forces acting in a crane. The following note does not offer a solution of the problem, but contains a proof by Mr. A. L. Jones that there is at most one position of equilibrium when the forces are all of one sign, i.e. either all tensions or all thrusts. This is followed by an investigation of the total number of solutions, real and imaginary, for all combinations of thrusts and tensions, when the magnitude only of each force, and not its sign, is given. This number is surprisingly large. It would of course be of more practical interest to find the number of real solutions for each particular combination of thrusts and tensions, but I have only succeeded in doing this when the given points are in a straight line, and in one or two other special cases. The results for these are stated without proof at the end of the note.

When the forces are all of one sign the single real position of equilibrium could be approximately found experimentally by means of threads, all knotted together at one end, which are passed through small smooth rings at the given points, and carry at their free ends weights proportional to the given forces. The position taken by the knot will then be the position of equilibrium.

Mr. Jones' proof (for forces all of one sign) depends on the simple lemma that two equal positive forces in the directions $P A, A Q$ have a resultant whose direction makes an acute angle with the direction of $P Q$. For the resultant $A R$ bisects the angle $Q A S$, formed by $A Q$ and $P A$ produced; and the line through $A$ in the direction $P Q$ makes with $A R$ an angle less than half $Q A S$, that is, an acute angle. The resultant cannot vanish unless $A$ is on the line $P Q$ and external to $P Q$; and the lemma holds if $A$ is internal to $P Q$.

Suppose that $P$ is a point such that given positive forces 
$t_{1}, t_{2}, \ldots, t_{n}$ acting from $P$ towards given points $A_{1}, A_{2}, \ldots, A_{n}$ are in equilibrium, and that if possible a second point $Q$ exists for which the same is true. Then the systern of forces $t_{1}, t_{2} \ldots, t_{n}$ along $P A_{1}, P A_{2}, \ldots, P A_{n}$, and the system of forces $t_{1}, t_{2}, \ldots, t_{n}$ along $A_{1} Q, A_{2} Q, \ldots, A_{n} Q$, are in equilibrium separately, and therefore in equilibrium jointly. But $t_{r}$ along $P A_{r}$ and $t_{r}$ along $A_{r} Q$ have a resultant which makes an acute angle with $P Q$; and the two systems are therefore equivalent to a single system of positive forces all of which make acute angles with $P Q$. This system cannot be in equilibrium unless all the forces vanish; and this cannot happen unless $A_{1}, A_{2}, \ldots, A_{n}$ are collinear, in which case there is either no position of equilibrium or an infinite number of such positions. If the given points are not collinear there can only be one real position of equilibrium at most.

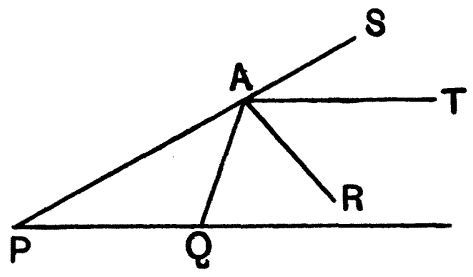

If forces $t_{2}, t_{3}, \ldots, t_{n}$ along $A_{1} A_{2}, A_{1} A_{3}, \ldots, A_{1} A_{n}$ have a resultant $t_{1}$, then $A_{1}$ is the position of equilibrium. The necessary and sufficient conditions that there may be a position of equilibrium other than $A_{1}, A_{2}, \ldots, A_{n}$ are that the resultant of the forces $t_{1}, \ldots, t_{r-1}, t_{r+1}, \ldots t_{n}$ along $A_{r} A_{1}, \ldots A_{r} A_{r-1}, A_{r} A_{r+1}, \ldots$ $A_{r} A_{n}$ should be greater than $t_{r}$, for all values of $r$ from 1 to $n$. For if these $n$ conditions hold, and the particle be placed at any one of the points $A_{1}, A_{2}, \ldots, A_{n}$, it will be drawn away by the forces which act upon it; and the forces cannot send the particle away to infinity, if they are tensions. For tensions the position of equilibrium is stable, and for thrusts unstable.

A slight extension of the above proof shows that if at one of the given points, say at $A_{1}$, the resultant of the forces $t_{2}, t_{3}, \ldots, t_{n}$ along $A_{1} A_{2}, A_{1} A_{3}, \ldots, A_{1} A_{n}$ is equal to or less than $t_{1}$, then for any other point $A_{r}$ the resultant of a like set of forces is greater than $t_{r}$. From this it follows that $n-1$ of the $n$ conditions mentioned above hold of necessity.

We will now consider the problem analytically and find the total number of solutions, real and imaginary, when the absolute magnitudes only of the forces are given. We consider first the case in which the given points are in a plane. Let $a_{r}, b_{r}$ be the rectangular coordinates of $A_{r}(r=1,2, \ldots n)$, and $x, y$ the coordinates of the particle $P$, which is in equilibrium under the system of forces $t_{1}, t_{2}, \ldots t_{n}$ along $P A_{1}, P A_{2} \ldots P A_{n}$. The equations for equilibrium, by resolving along the axes, are 
where

$$
\begin{aligned}
& \frac{t_{1}\left(x-a_{1}\right)}{r_{1}}+\frac{t_{2}\left(x-a_{2}\right)}{r_{2}}+\ldots+\frac{t_{n}\left(x-a_{n}\right)}{r_{n}}=0, \\
& \frac{t_{1}\left(y-b_{1}\right)}{r_{1}}+\frac{t_{2}\left(y-b_{2}\right)}{r_{2}}+\ldots+\frac{t_{n}\left(y-b_{n}\right)}{r_{n}}=0,
\end{aligned}
$$

Assuming $r_{1}, r_{2}, \ldots r_{n}$ to be always positive, ${ }^{*}$ we cannot in general find the solutions of these equations without at the same time finding the solutions for all the $2^{n-1}$ combinations of thrusts and tensions among the given forces. We shall, however, assume that $r_{1}, r_{2}, \ldots r_{n}$ are capable of either sign, while $t_{1}, t_{2}, \ldots t_{n}$ remain invariably positive, as this gives us the same system of equations, and leaves the values of the unknowns $x, y, r_{1}, r_{2}, \ldots r_{n}$ unrestricted. In order to discuss the infinite (as well as the finite) solutions we introduce another unknown $u$ so as to make the equations homogeneous. Then the system of equations for the ratios of $u, x, y, r_{1}, \ldots r_{n}$ is

$$
\begin{array}{r}
\frac{t_{1}\left(x-a_{1} u\right)}{r_{1}}+\ldots+\frac{t_{n}\left(x-a_{n} u\right)}{r_{n}}=0, \ldots \\
\frac{t_{1}\left(y-b_{1} u\right)}{r_{1}}+\ldots+\frac{t_{n}\left(y-b_{n} u\right)}{r_{n}}=0, \ldots \\
\left(x-a_{1} u\right)^{2}+\left(y-b_{1} u\right)^{2}=r_{1}^{2}, \\
\left(x-a_{n} u\right)^{2}+\left(y-b_{n} u\right)^{2}=r_{n}{ }^{2} .
\end{array}
$$

Equations (1), (2) are written in a fractional form so as to be less cumbersome, but can be at once made integral by multiplying by $r_{1} r_{2} \ldots r_{n}$. Thus there are two equations of degree $n$, and $n$ equations of degree 2 , and the total number of solutions, taking account of infinite and multiple solutions, is $n^{2} 2^{n}$. There are, however, a large number of irrelevant solutions which do not satisfy the conditions of the problem; their number must be found and subtracted from $n^{2} 2^{n}$ in order to tind the true number of solutions of the problem.

In particular, all infinite solutions (those in which $u=0$ ) are irrelevant, and must be rejected; it will be found that there are only two different infinite solutions, but that each is multiple to a high degree. In addition some multiple and non-multiple finite solutions are also irrelevant. In general the relevant solutions are all non-multiple and finite.

* The function $t_{1} r_{1}+t_{2} r_{2}+\ldots+t_{n} r_{n}$ is the potential of the system of forces, and has a stationary value at a position of equilibrium $P$. The curve of the family

$$
t_{1} r_{1}+t_{2} r_{2}+\ldots+t_{n} r_{n}=\text { constant }
$$

which passes through $P$, has a node at $P$; and a node is a position of equilibrium. If two positions of equilibrium move up to coincidence the node becomes a cusp. 
The chief difficulty is to find the multiplicity of the multiple solutions. To do this we make use of a principle which is seldom applied: In a given system of $n$ non-homogeneous equations for $n$ unknowns $x_{1}, \ldots x_{n}$ the multiplicity of the solution

$$
x_{1}=x_{2}=\ldots=x_{n}=0
$$

is the product of the degrees of the terms of lowest degree in the equations, provided that these lowest terms when equated to zero have no proper solution, that is, no solution other than $x_{1}=x_{2}=\ldots=x_{n}=0$. Again the multiplicity of the solution $x_{1}=a_{1}$, $x_{2}=a_{2}, \ldots x_{n}=a_{n}$ is found by the same rule after substituting $x_{1}+a_{1}, \ldots x_{n}+a_{n}$ for $x_{1}, \ldots x_{n}$ in all the equations. Finally the rule is applied to a system of homogeneous equations by making them non-homogeneous, by putting a non-vanishing unknown, of which there must be at least one in any proper solution, equal to unity.

The rule can only be applied in what is called the "simple case," viz. when the equations can be so written that the lowest. terms when equated to zero have no proper solution. For example, the multiplicity of the solution $x_{1}=x_{2}=0$ in the equations $x_{1}-x_{2}+x_{1}^{2}=0, x_{1}^{2}-x_{2}^{2}+2 x_{1}^{2} x_{2}=0$ is 4 ; for, on multiplying the first by $x_{1}+x_{2}-x_{1}^{2}$, and subtracting from the second, we get $x_{1}^{4}=0$, which may be taken in place of the second equation. The equations being written in any definite order, each equation may be modified as above by means of previous equations, but not by means of succeeding equations, since such modification does not affect the number or the multiplicity of the finite solutions. In the case of the equations $x_{1}{ }^{2}-x_{2}{ }^{2}+x_{1}{ }^{3}=0$, $x_{1}^{3}-x_{2}^{3}+x_{2}^{4}=0$, the proviso cannot be fulfilled and the rule cannot be applied. This example belongs to what is called the "general case"; but all the solutions of the system of equations (1) to $(n+2)$ come under the "simple case."

We can now proceed to find all the irrelevant solutions of the equations (1) to $(n+2)$. Consider first the infinite solutions. On putting $u=0$, the equations become $x \Sigma \frac{t}{r}=y \Sigma \frac{t}{r}=0$, $x^{2}+y^{2}=r_{1}^{2}=\ldots=r_{n}{ }^{2}$. Hence either $x=y=0$, which gives no proper solution; or else $\Sigma \frac{t}{r}=0$, which requires all the $r$ 's to vanish, unless one of the expressions $t_{1} \pm t_{2} \pm \ldots \pm t_{n}$ vanishes, which we shall assume not to be the case. Thus there are only two different infinite solutions, viz. $u=r_{1}=r_{2}=\ldots=r_{n}=0$, $x= \pm ı$.

To find the multiplicity of the infinite solution corresponding to $x+\iota y=0$, put $x+\iota y=v$ and $x-\iota y=1$, thus making the system non-homogeneous with $v, u, r_{1}, \ldots r_{n}$ as unknowns, and

$$
u=v=r_{1}=\ldots=r_{n}=0
$$


as the solution whose multiplicity is required. Now from equations (3), (4) we can express $u$ and $v$ as quadratic functions of $u, v, r_{1}, r_{2}$, and by substituting them in equations $(5)$ to $(n+2)$, the lowest terms in these become of degree 2. Also (1), (2) become $(v+1) \Sigma \frac{t}{r}=2 u \Sigma \frac{a t}{r}, \quad(v-1) \Sigma \frac{t}{r}=2 \iota u \Sigma \frac{b t}{r}$, of which the second may be replaced by $v \Sigma \frac{t}{r}=u \Sigma \frac{(a+b) t}{r}$, which is of degree $n+1$ when made integral, and when for $u, v$ are substituted the quadratic functions to which they are equal. The product of the degrees of the lowest terms in the equations is now

$$
(n-1)(n+1) 2^{n-2} \text {. }
$$

This is the multiplicity of each of the infinite solutions. The number of finite solutions is therefore

$$
n^{2} 2^{n}-\left(n^{2}-1\right) 2^{n-1}=\left(n^{2}+1\right) 2^{n-1} .
$$

As regards these we may put $u=1$, since $u=1=0$.

The irrelevant finite solutions are of two kinds. There are $2^{n-1}$ solutions for which $x=a_{p}, y=b_{p}, r_{p}=0$; for these values satisfy equations (1), (2), $(p+2)$, and the other equations have $2^{n-1}$ solutions. Each of these solutions is of multiplicity 2, owing to equation $(p+2)$. This accounts for $n 2^{n}$ irrelevant solutions. Again there are $2^{n-1}$ finite solutions for which $r_{p}=r_{q}=0$; for these values satisfy (1), (2), and leave all the other equations quadratic except one of the equations $(p+2)$, $(q+2)$; which give a linear equation by subtraction.* This accounts for $\frac{1}{2} n(n-1) 2^{n-1}$ irrelevant solutions. Assuming that this exhausts the number of irrelevant solutions we find the number of relevant solutions to be

$$
\left(n^{2}+1\right) 2^{n-1}-n 2^{n}-n(n-1) 2^{n-2}=(n-1)(n-2) 2^{n-2} .
$$

The number of solutions for $x, y$ is only half of this, since to each solution $x, y, r_{1}, r_{2}, \ldots r_{n}$ there corresponds another $x, y$, $-r_{1},-r_{2}, \ldots-r_{n}$, in which $x, y$, have the same values. The final result is that the total number of solutions of the problem for $n$ co-planar forces is ${ }_{n-1} C_{2} 2^{n-2}$. I have verified by other methods that this number is correct when $n=3,4$, or 5 , which goes to show that the method of exhaustion followed above is complete. The number is the total number of solutions, real and imaginary, for all the $2^{n-1}$ combinations of thrusts and tensions among the given forces. The average number of solutions for each combination is therefore $\frac{1}{4}(n-1)(n-2)$.

When the given points are co-planar there is a case of exception in which the number of solutions is infinite. This occurs when

\footnotetext{
* This diminution to half the normal number of solutions is accounted for by the fact that the other half are at infinity. The infinite solutions have been previously considered separately.
} 
the points all lie on a circle, and a point on the circle, other than one of the given points, is a position of equilibrium. In that case every point on the circle is a position of equilibrium. Also (when the points are on a circle) if there is any real position $P$ of equilibrium outside the plane, then every point on a certain .circle through $P$ is a position of equilibrium. This circle and the given circle lie in perpendicular planes, and each cuts the plane of the other in a pair of inverse points.

A similar method can be applied to find the total number of solutions of the problem for $n$ given points in ordinary space. The process is longer and more difficult, and we shall give only a short sketch of it.

In this case we have $n+3$ equations for the ratios of the $n+4$ unknowns $u, x, y, z, r_{1}, r_{2}, \ldots r_{n}$, viz.

$$
\begin{aligned}
& \frac{t_{1}\left(x-a_{1} u\right)}{r_{1}}+\ldots+\frac{t_{n}\left(x-a_{n} u\right)}{r_{n}}=0, \\
& \frac{t_{1}\left(y-b_{1} u\right)}{r_{1}}+\ldots+\frac{t_{n}\left(y-b_{n} u\right)}{r_{n}}=0, \\
& \frac{t_{1}\left(z-c_{1} u\right)}{r_{1}}+\ldots+\frac{t_{n}\left(z-c_{n} u\right)}{r_{n}}=0, \\
& \left(x-a_{1} u\right)^{2}+\left(y-b_{1} u\right)^{2}+\left(z-c_{1} u\right)^{2}=r_{1}^{2}, \\
& \left(x-a_{n} u\right)^{2}+\left(y-b_{n} u\right)^{2}+\left(z-c_{n} u\right)^{2}=r_{n}{ }^{2} .
\end{aligned}
$$

These equations have a singly infinite system of solutions, together with a finite number of other solutions which include all the relevant solutions. In geometrical language equations (2) to $(n+3)$ have as their complete intersection a composite curve of degree $n^{2} 2^{n}$, part of which lies in (1), and the remaining part, which we shall call the remainder curve, cuts (1) in a finite number of points, some giving relevant and some irrelevant solutions. We must first find the degree of the remainder curve.

Part of the intersections of (2) to $(n+3)$ consists of an infinity curve of degree 2 , which lies in (1), viz. the curve

$$
u=r_{1}=r_{2}=\ldots=r_{n}=0, x^{2}+y^{2}+z^{2}=0 .
$$

For, on putting $u=0$, we have

$$
y \Sigma \frac{t}{r}=z \Sigma \frac{t}{r}=0, x^{2}+y^{2}+z^{2}=r_{1}^{2}=r_{2}^{2}=\ldots=r_{n}^{2} .
$$

Hence either $y=z=0$, giving $2^{n}$ points, none of which lie in (1); or $\Sigma \frac{t}{r}=0$, which requires all the $r$ 's to vanish, assuming that no expression of the form $t_{1} \pm t_{2} \pm \ldots \pm t_{n}$ vanishes. This gives the infinity curve. To find its multiplicity in the intersection of (2) to $(u+3)$ we put $z=1$, and change $x, y$ to $x+x_{1}$, 
$y+y_{1}$, where $x_{1}^{2}+y_{1}^{2}+1=0$. From equations (4), (5) we can then express $u$ and $x x_{1}+y y_{1}$ as quadratic functions of $u, x, y$, $r_{1}, r_{2}$; and by substituting them in $(6)$ to $(n+3)$ the lowest terms in these last become of degree 2. Also equations (2) (3) become $\left(y+y_{1}\right) \Sigma \frac{t}{r}=u \Sigma \frac{b t}{r}, \Sigma \frac{t}{r}=u \Sigma \frac{c t}{r}$, the former of which may be changed to $u \Sigma \frac{b t}{r}=\left(y+y_{1}\right) u \Sigma \frac{c t}{r}$, whose lowest terms are of degree $n+1$, when we substitute for $u$ the quadratic function to which it is equal. The infinity curve is therefore of multiplicity $(n+1)(n-1) 2^{n-2}$, and counts as of degree $\left(n^{2}-1\right) 2^{n-1}$, in the intersection of $(2)$ to $(n+3)$. The complete finite intersection of $(2)$ to $(n+3)$ is therefore a curve of degree

$$
n^{2} 2^{n}-\left(n^{2}-1\right) 2^{n-1}=\left(n^{2}+1\right) 2^{n-1} \text {. }
$$

This finite curve includes $\frac{1}{2} n(n-1)$ curves which lie in (1) viz. those for which any two of the $r$ 's vanish. On putting $u=1$ and $r_{1}=r_{2}=0$, equations (4), (5) are equivalent to one quadratic and one linear equation, while $(6)$ to $(n+3)$ are quadratic. Hence each of the $\frac{1}{2} n(n-1)$ curves is of degree $2^{n-1}$, and the remainder curve is of degree

$$
\left(n^{2}+1\right) 2^{n-1}-n(n-1) 2^{n-2}=\left(n^{2}+n+2\right) 2^{n-2},
$$

which cuts $(1)$ in $n\left(n^{2}+n+2\right) 2^{n-2}$ points altogether.

All the points in which the remainder curve cuts (1) on the infinity curve and the $\frac{1}{2} n(n-1)$ curves give irrelevant solutions, and also a few other points. The remainder curve meets infinity $(u=0)$ in $\left(n^{2}+n+2\right) 2^{n-2}$ points, of which only $2^{n}$ (corresponding to $y=z=0$ ) are not on the infinity curve. The other points lie on the infinity curve; but at each of these points the remainder curve touches $u=0$, so that it has only $\left(n^{2}+n-2\right) 2^{n-3}$ points of intersection with the infinity curve. At each of the $\left(n^{2}+n-2\right) 2^{n-3}$ points the remainder curve meets (1) in a point of multiplicity $n+1$. This accounts for $(n+1)\left(n^{2}+n-2\right) 2^{n-3}$ irrelevant infinite solutions.

At any finite point at which three of the $r$ 's vanish, the complete intersection of $(2)$ to $(n+3)$ has four branches, three of which belong to curves for which two of the $r$ 's vanish, and the fourth to the remainder curve. There are $2^{n-2}$ such finite points at which any one set of three $r$ 's vanish, and at each the remainder curve meets (1) in a point of multiplicity 2. This accounts for $\frac{1}{6} n(n-1)(n-2) 2^{n-1}$ irrelevant finite solutions.

The remainder curve also cuts the curve corresponding to $r_{p}=r_{q}=0$ in $2^{n-1}$ finite points at which no other $r$ vanishes, viz. the points for which $\frac{y-b_{p}}{z-c_{p}}=\frac{y-b_{q}}{z-c_{q}}=\frac{b_{p}-b_{q}}{c_{p}-c_{q}}$. This accounts for $\frac{1}{2} n(n-1) 2^{n-1}$ irrelevant solutions. 
Also the remainder curve meets (1) in $2^{n-1}$ points corresponding to $x=a_{p}, y=b_{p}, z=c_{p}, r_{p}=0$, each of which is of multiplicity 2 . This accounts for $n 2^{n}$ irrelevant solutions.

Hence we find the number of relevant solutions to be

$$
\begin{aligned}
& n\left(n^{2}+n+2\right) 2^{n-2}-(n+1)\left(n^{2}+n-2\right) 2^{n-3} \\
& \quad \quad-\frac{1}{6} n(n-1)(n-2) 2^{n-1}-n(n-1) 2^{n-2}-n 2^{n} \\
& =\frac{1}{6}(n+3)(n-1)(n-2) 2^{n-2} \\
& \quad=\frac{1}{6}(n-1)(n-2)(n-3) 2^{n-2}+(n-1)(n-2) 2^{n-2} .
\end{aligned}
$$

The number of solutions for $x, y, z$ is half of this.

Hence the total number of solutions of the problem for $n$ forces in ordinary space is ${ }_{n-1} \mathrm{C}_{2} 2^{n-2}+{ }_{n-1} \mathrm{C}_{3} 2^{n-3}$.

This result is correct also if the given points are all in one plane, for then the total number of solutions in the plane is ${ }_{n-1} C_{2} 2^{n-2}$, and out of the plane ${ }_{n-1} C_{3} 2^{n-3}$. This enables us to guess the answer to the problem for $n$ forces in space of $m$ dimensions. We may in fact assume the answer to be

$$
{ }_{n-1} C_{2} 2^{n-2}+{ }_{n-1} C_{3} 2^{n-3}+\ldots+{ }_{n-1} C_{m} 2^{n-m} .
$$

For $n+1$ forces in space of $n$ dimensions the number of solutions is $2\left(3^{n}-\overline{n+2} 2^{n-1}\right)$, a result which can be verified in other ways.

As regards the number of real solutions, when the given points are not collinear, I can only mention two results: (i) that for four points in ordinary space the fourteen solutions can all be real ; (ii) that for $n+1$ points in space of $n$ dimensions, when the distance between every pair of points is the same, and the forces are all equal, there is one and only one real solution for each combination of thrusts and tensions. If, however, $n+1$ is even, the solutions corresponding to an equal number of thrusts and tensions are at infinity and of multiplicity 3 .

In the case of a tetrahedron of which the base is equilateral, and the three faces have the same inclination $2 \alpha$ to the base, if the force to (or from) the vertex is $t_{0}$, and to (or from) each of the base angles is $t$, there will be fourteen real solutions provided $t_{0} / t$ lies between 1 and $3 \sin \alpha\left(1-3 \sin ^{2} \alpha \cos ^{2} \alpha\right)^{\frac{1}{2}}$. (Thus for a rectangular tetrahedron $t_{0} / t$ must lie between $97 \check{0} \ldots$ and 1 ). When all four forces are tensions, or when three are tensions and one a thrust, there is one solution in each case, and when two are tensions and two are thrusts there are three solutions in each case.

When the given points are collinear the number of real positions of equilibrium in any plane through the line of collinearity can be stated completely, provided no expression of the form $t_{1} \pm t_{2} \pm \ldots \pm t_{n}$ vanishes. Let $A_{1}, A_{2}, \ldots A_{n}$ be the given points in order, and $t_{1}, t_{2}, \ldots t_{n}$ the forces (given in magnitude and sign) directed towards them. As the sign of one force may be chosen either positive or negative, we assume that $t_{1}$ is positive. Then the number of real positions of equilibrium on each side of the 
line is the excess of the number of positive maxima over the number of positive minima in the series of quantities

where

$$
\begin{aligned}
& \left(-S_{n}\right), S_{1}, S_{2}, \ldots S_{n},\left(-S_{1}\right) \text {, } \\
& S_{1}=-t_{1}+t_{2}+\ldots+t_{n}=\Sigma t-2 t_{1} \text {, } \\
& S_{2}=-t_{1}-t_{2}+\ldots+t_{n}=S_{1}-2 t_{2} \text {, } \\
& S_{n}=-t_{1}-t_{2}-\ldots-t_{n}=S_{n-1}-2 t_{n}
\end{aligned}
$$

By a maximum (or minimum) quantity is meant one which is greater (or less) than each of the adjacent quantities. The rule is applied to $S_{1}, S_{2}, \ldots S_{n}$ only, $S_{1}$ and $S_{n}$ being counted as minima if less than the adjacent quantity, but not as maxima if greater than the adjacent quantity, since $S_{1}<-S_{n}, S_{n}<-S_{1}$.

Thus if there are $2 n+1$ equal forces alternately pushing and pulling, there are $n$ real positions of equilibrium on each side of the line.

If $t_{1}, t_{2}, \ldots t_{n}$ are the absolute magnitude of the forces, and positive, the total number of real positions of equilibrium for all possible combinations of thrust and tension is the excess of the total number of positive signs over the total number of negative signs in all the expressions $-t_{1} \pm t_{2} \pm \ldots \pm t_{n}$ which have positive values. This result (like the last) is independent of the distances between the given points, and (unlike the last) remains unchanged if the order of the points is changed.

The greatest possible number of real positions of equilibrium is found by subtracting each coefficient in the expansion of $(1+x)^{n-1}$ from the greatest coefficient, and adding all the results. This is counting the solutions on both sides of the line; and may be compared with the total number of solutions, real and imaginary, viz. $(n-1)(n-2) 2^{n-3}$. The ratio is roughly that of 3 to $n^{\frac{3}{2}}$.

F. S. Macaulay.

\section{REVIEWS.}

The Theory of Sets of Points. By W. H. Young, M.A., Sc.D., and Grace Chisholm Young, Phil. Doc. (Gött.). (Cambridge : at the University Press. 1906. Pp. xii +316 .)

The theory of aggregates may roughly be divided into two parts: the one, which arose out of the profound study of integration, is associated with the names of Riemann, Hankel, Smith, Harnack, P. du Bois-Reymond, Lebesgue, and Young; while the other, which arose from the need for a secure basis of the theory of analytic functions, has, as characteristics, Weierstrass' and Cantor's theories of irrationals, Cantor's theory of derivatives of point aggregates, Cantor's theory of the 'powers' (Mächtigkeiten) of aggregates, Cantor's theory of transfinite numbers, and the modern developments of this last theory. The two parts are by no means disconnected; thus, Cantor has greatly 